\title{
Development of a competitive chemiluminescence immunoassay using a monoclonal antibody recognizing 3B of foot-and-mouth disease virus for differentiating infected from vaccinated animals
}

\author{
Wei Liu ${ }^{1}$, Guanglei Zhang ${ }^{1}$, Sicheng Yang ${ }^{1}$, Junhui Li ${ }^{1}$, Sude Ge ${ }^{1}$, Huihui Yang ${ }^{1}$, Jun-Jun \\ Shao $^{2}$, and Hui-Yun Chang ${ }^{1}$ \\ ${ }^{1}$ Affiliation not available \\ ${ }^{2}$ Lanzhou Veterinary Research Institute
}

January 24, 2021

\begin{abstract}
Foot-and-mouth disease (FMD) is a devastating animal disease. Differentiation of infected from vaccinated animals (DIVA) is very important for confirming suspected cases, evaluating the prevalence of infection, certifying animals for trade and controlling the disease. In this study, a competitive chemiluminescence immunoassay (3B-cCLIA) for DIVA was developed for the rapid detection of antibodies against non-structural proteins (NSPs) in different species of livestock animals using monoclonal antibody 9E2 as a competitive antibody that recognizes NSP 3B, which only needs to be washed once and takes 15 min. The cut-off (50\%), diagnosis sensitivity $(97.20 \%, 95.71 \%$, and $96.15 \%)$ and diagnosis specificity $(99.51 \%, 99.43 \%$, and 98.36$)$ of the assay were estimated by testing a panel of known background sera from swine, cattle and sheep. The accuracy rate of 3B-cCLIA was also validated and compared with that of two commercial diagnostic kits. The early diagnostic performance showed that antibodies to NSPs occurred later (approximately 1-2 days) than antibodies to structural proteins. Furthermore, NSP antibodies present in animals vaccinated multiple times (false-positive), especially in cattle and sheep, were confirmed, and the false-positive rate increased with the number of vaccinations. These results indicated that 3B-cCLIA is suitable to rapidly detect antibodies against FMDV NSP 3B in a wide range of species for DIVA.
\end{abstract}

Development of a competitive chemiluminescence immunoassay using a monoclonal antibody recognizing $3 \mathrm{~B}$ of foot-and-mouth disease virus for differentiating infected from vaccinated animals

Running Title: Competitive CLIA for detection of 3B antibodies

Wei Liu, Guanglei Zhang, Sicheng Yang, Junhui Li, Sudan Ge, Huihui Yang, Junjun Shao\#, Huiyun Chang\#

State Key Laboratory of Veterinary Etiological Biology, National Foot-and-Mouth Diseases Reference Laboratory, Lanzhou Veterinary Research Institute, Chinese Academy of Agricultural Sciences, Lanzhou, Gansu Province, China

\#Address correspondence to Huiyun Chang (changhuiyun@caas.cn)and Junjun Shao (shaojunjun@caas.cn)

\section{Summary}

Foot-and-mouth disease (FMD) is a devastating animal disease. Differentiation of infected from vaccinated animals (DIVA) is very important for confirming suspected cases, evaluating the prevalence of infection, certifying animals for trade and controlling the disease. In this study, a competitive chemiluminescence immunoassay (3B-cCLIA) for DIVA was developed for the rapid detection of antibodies against non-structural 
proteins (NSPs) in different species of livestock animals using monoclonal antibody 9E2 as a competitive antibody that recognizes NSP 3B, which only needs to be washed once and takes $15 \mathrm{~min}$. The cut-off (50\%), diagnosis sensitivity $(97.20 \%, 95.71 \%$, and $96.15 \%)$ and diagnosis specificity $(99.51 \%, 99.43 \%$, and 98.36) of the assay were estimated by testing a panel of known background sera from swine, cattle and sheep. The accuracy rate of 3B-cCLIA was also validated and compared with that of two commercial diagnostic kits. The early diagnostic performance showed that antibodies to NSPs occurred later (approximately 1-2 days) than antibodies to structural proteins. Furthermore, NSP antibodies present in animals vaccinated multiple times (false-positive), especially in cattle and sheep, were confirmed, and the false-positive rate increased with the number of vaccinations. These results indicated that 3B-cCLIA is suitable to rapidly detect antibodies against FMDV NSP 3B in a wide range of species for DIVA.

Keywords: chemiluminescence immunoassay, diagnosis, foot-and-mouth disease virus, monoclonal antibody, non-structural protein

\section{INTRODUCTION}

Foot-and-mouth disease (FMD) is a highly infectious and economically devastating viral disease of clovenhoofed animals. The causative agent, FMD virus (FMDV), has a positive-sense and single-stranded RNA that codes for four structural proteins (SPs: VP4, VP2, VP3 and VP1) and 10 non-structural proteins (NSPs: L, 2A, 2B, 2C, 3A, 3B, 3C, 3D, 3AB and 3ABC) (Jaworski et al., 2011; W. Liu et al., 2019; Z. Z. Liu et al., 2018).

To date, slaughtering infected and contacted animals together with prohibiting the import of animal and animal products from FMD endemic countries have been adopted to prevent the disease in FMD-free nations. Considering the economic costs, a vaccination policy was adopted for control and eradication of the disease in endemic countries (Sharma et al., 2012; Uttenthal et al., 2010). However, vaccination with inactivated vaccines raises other issues, such as the differentiation of FMDV-infected from vaccinated animals (DIVA) and the production of carrier animals to shed the virus (Sharma et al., 2012; Uttenthal et al., 2010). Therefore, the detection of antibodies against NSPs is the preferred diagnostic method for DIVA and helps to identify subclinical infections, evaluate the prevalence of infection and control the disease (Lu et al., 2010) because most NSPs are removed from the inactivated vaccine by a series of purification methods during production (Fu et al., 2014; W. Liu et al., 2019).

Thus far, there are three main diagnostic types of detecting antibodies against NSPs for DIVA. One is indirect ELISA or LFIA based on recombinant NSPs, such as recombinant fusion protein of 2B (Biswal, Jena, Mohapatra, Bisht, \& Pattnaik, 2014), 2C (Y. Gao, Sun, \& Guo, 2016; Mackay et al., 1998; Meyer et al., 1997), 3A (Biswal, Ranjan, \& Pattnaik, 2016; Kumar, Sharma, \& Kakker, 2007), 3B (A. K. Mohapatra, Mohapatra, Pandey, Sanyal, \& Pattnaik, 2014), 3AB (Jaworski et al., 2011; J. K. Mohapatra, Pandey, Sanyal, \& Pattnaik, 2011), 2C3AB (Lu et al., 2010; Wu et al., 2011), or 3ABC (Brocchi et al., 2006; Bruderer et al., 2004; De Diego, Brocchi, Mackay, \& De Simone, 1997; Hosamani et al., 2015; Lu et al., 2007; Robiolo et al., 2006). In particular, 3ABC is the most appropriate indicator because of its high immunogenicity and its relatively low abundance in infected cell lysates (W. Liu et al., 2019; Z. Liu et al., 2017; Lu et al., 2010; Robiolo et al., 2006; Van Dreumel et al., 2015). Another is ELISA based on peptides or epitopes derived from NSPs 2B, 2C, 3A, and 3B (M. Gao et al., 2012; Höhlich, Wiesmüller, Haas, et al., 2003; Höhlich, Wiesmüller, Schlapp, et al., 2003; Inoue et al., 2006; Oem et al., 2005; Shen et al., 1999). Both indirect ELISAs based on recombinant NSPs or epitopes that use species-specific conjugates make simultaneous examination of sera from different species difficult (Sørensen, de Stricker, Dyrting, Grazioli, \& Haas, 2005). Block or competitive ELISA using polyclonal antibodies (Chung, Sorensen, Liao, Yang, \& Jong, 2002; Sørensen et al., 1998; Van Dreumel et al., 2015) or monoclonal antibodies (mAbs) (Fu et al., 2017; Fu et al., 2014; Gelkop et al., 2018; Muller et al., 2010; Oem et al., 2007; Sharma et al., 2012; Sørensen et al., 2005) has been widely used because it can be used for each species that is susceptible to FMD.

In recent years, chemiluminescence immunoassay (CLIA) has gained increasing attention in many fields, 
including clinical diagnosis, food analysis, and environmental monitoring, because of its high sensitivity, high signal-to-noise ratio, wide linear ranges and low cost (Wang, Jie, Zong, Jie, \& Huang-Xian, 2012). In the DIVA test, indirect CLIAs based on recombinant NSPs 2C and 3ABC (Z. Liu et al., 2017), as well as two epitopes located in 3A and 3B (Z. Z. Liu et al., 2018), were developed.

In this study, we used mAb 9E2, which recognizes NSP 3B, to develop a competitive CLIA (termed 3BcCLIA) for the rapid detection of NSP antibodies, which can be used for a wide range of species. The assay was evaluated with sera collected from naïve, vaccinated and infected animals, and the accuracy rate was validated and compared with that of two commercial kits.

\section{MATERIALS AND METHODS}

\subsection{Serum samples}

Serum samples from naïve animals: Serum samples from clinically healthy and unvaccinated animals, including 310, 175, and 61 samples from swine, cattle, and sheep, respectively, were collected and tested using liquid-phase blocking ELISA of FMDV O (O-LPBE) and A-LPBE (presented negative results; titer, < 1:4). These samples were utilized to estimate the cut-off value and diagnostic specificity (Dsp).

Serum samples from infected animals: A total of 107 serum samples were collected from swine infected with FMDV O/Mya98 or A/GDMM/2013 at 7-25 days post infection (dpi) in the Animal Biological Safety Level 3 (ABSL-3) Laboratory at Lanzhou Veterinary Research Institute (Lanzhou, China); 70 serum samples were collected from cattle infected with FMDV (O/Mya98 or A/GDMM/2013) at 8-20 dpi; 52 serum samples were collected from sheep during the early infection period. All of these samples were used to estimate the cut-off value and diagnostic sensitivity (Dsn). In addition, a total of 32 sera collected from four unvaccinated control swine experimentally challenged with FMDV O/Mya98 at 0 dpi and 2-8 dpi were used to compare the early diagnostic performance of 3B-cCLIA and two commercial diagnostic kits (3ABC monoclonal antibody blocking ELISA (3ABC-bELISA) and PrioCHECK FMDV NSP ELISA) and identify seroconversion to FMDV SP and NSP.

Serum samples from vaccinated animals: One hundred serum samples were collected at 21 days post vaccination (dpv) from swine vaccinated with FMDV O univalent multiple-epitope recombinant vaccine developed by our research group. These samples were utilized to estimate the cut-off value and diagnostic specificity (Dsp). In addition, 120 serum samples were collected from sows vaccinated 3-15 times with commercial O/A divalent inactivated vaccines; 129 serum samples were collected from dairy cows vaccinated with commercial $\mathrm{O} /$ A divalent inactivated vaccines every four months for a total of 2,5 or 10 vaccinations. Seventy-seven serum samples were collected from 15 sheep vaccinated 1-3 times with laboratory-made FMDV O/Mya98/BY/2010, FMDV A/AF72, or FMDV Asia 1/JS05 univalent inactivated vaccine. These samples were used to evaluate the diagnostic performances of 3B-cCLIA and two commercial diagnostic kits and verified the false-positive phenomenon with animals vaccinated multiple times.

Serum samples collected in the field: 173 field sera collected from swine suspected of FMDV infection during 2010-2018. These sera were used to compare the coincidence rates between 3B-cCLIA and two commercial diagnostic kits.

Serum samples from other virus-infected swine: One serum sample from senecavirus A (SVA)-infected swine, one from classical swine fever virus (CSFV)-infected swine, one from porcine reproductive and respiratory syndrome virus (PRRSV)-infected swine, one from porcine parvovirus (PPV)-infected swine, and two from porcine circovirus type 2 (PCV2)-infected swine were examined in this study.

Control sera: The serum sample derived from swine infected with FMDV O/Mya98 at 25 dpi served as the standard positive serum (P51). The percentage inhibition (PI) of the serum in the 3ABC-bELISA and PrioCHECK FMDV NSP ELISA was $92 \%$ and $93 \%$, respectively. The serum collected from clinically healthy swine with no immunization against FMD served as the standard negative serum sample (P734). The serum 
was tested with O-LPBE and A-LPBE (produced negative result; titer, < 1:4), 3ABC-bELISA (produced negative result; PI $=1 \%$ ), and PrioCHECK FMDV NSP ELISA (produced negative result; PI $=-7 \%$ ).

\subsection{Antigen and antibodies}

The 3ABC coding region of FMDV A/GDMM/2013 mutated at positions 46 aa and 163 aa was cloned into the pProEXHTB plasmid. The expression and purification of the recombinant $3 \mathrm{ABC}$ protein has been described (Z. Liu et al., 2017).

The mAbs against the $3 \mathrm{ABC}$ protein, designated $2 \mathrm{G} 5$ and $9 \mathrm{E} 2$, were obtained at our laboratory, and their minimally identified epitopes are "92 EYIEKA ${ }^{97 "}$ ", which is located in $3 \mathrm{~A}$, and "23EGPYAGPLE" ${ }^{31 ", ~ w h i c h ~ i s ~}$ located in 3B (W. Liu et al., 2019). MAbs 2G5 and 9E2 were largely produced by injecting hybridomas into the peritoneal cavities of BALB/c mice and purified by affinity protein G column chromatography. Then, the mAbs 2G5 and 9E2 were conjugated with horseradish peroxidase (HRP). The polyclonal antibody was obtained by inoculating rabbits with the purified $3 \mathrm{ABC}$ protein.

\subsection{Development of competitive CLIA using mAb against NSP 3B}

The conditions of 3B-cCLIA were optimized by checkerboard titration. Briefly, mAb 2G5 was coated onto 96-well white plates (Costar) at the concentrations of $1,0.5,0.25$, and $0.125 \mu \mathrm{g} / \mathrm{mL}$ in a $100-\mu \mathrm{L}$ volume and incubated overnight at $4{ }^{\circ} \mathrm{C}$. After washing, purified $3 \mathrm{ABC}$ protein was diluted to $0.5,0.25$, and $0.125 \mu \mathrm{g} / \mathrm{mL}$ in PBST (PBS containing 0.05\% Tween-20) and added to each well, and the plate was incubated for $1 \mathrm{~h}$ at $37{ }^{\circ} \mathrm{C}$. After three washes with PBST, $200 \mu \mathrm{L}$ of blocking buffer was added to each well and incubated for $2 \mathrm{~h}$ at $37{ }^{\circ} \mathrm{C}$. Then, standard positive serum (P51) and standard negative serum (P734) were serially diluted with dilution buffer ( $1 \%$ casein, $10 \%$ equine serum in PBST) at 1:2.5-1:20 dilutions, and $50 \mu \mathrm{L}$ of the serum was transferred to each well. Simultaneously, $50 \mu \mathrm{L}$ of HRP-conjugated mAb 9E2 (9E2-HRP) at concentrations of $0.08,0.04,0.02,0.01,0.005$, and $0.0025 \mu \mathrm{g} / \mathrm{mL}$ was added to each well, and the plate was incubated at ambient temperature. Finally, after five washes, $100 \mu \mathrm{L}$ of chemiluminescence (CL) substrate (KEY-BIO, Beijing, China), including $50 \mu \mathrm{L}$ of solution A (luminol and luminous enhancer) and $50 \mu \mathrm{L}$ of solution B (peroxide solution), were added. After $5 \mathrm{~min}$, the CL signals were measured using Varioskan lux (Thermo Scientific, USA). The optimum mAb 2G5 concentration, 3ABC protein concentration, serum dilution, 9E2-HRP concentration, and incubation time were determined on the basis of the ratios of CL values of standard negative serum to standard positive serum $(\mathrm{N} / \mathrm{P})$.

3B-cCLIA was performed under optimized condition. The PI was calculated using the following formula:

$\mathrm{PI} \%=(1-\mathrm{CLs} / \mathrm{CLn}) \times 100 \%$,

where CLs are the CL values of the test samples and CLn are the mean CL values of the standard negative serum control.

A mean CLn [?] 6,000,000 and mean PI of the standard positive control $>80 \%$ indicate assay validity.

Similarly, 3A+3B-cCLIA was developed, and detailed information is presented in the supplemental material.

\subsection{Cut-off value, Dsn, and Dsp}

The cut-off value of 3B-cCLIA was determined by testing 875 serum samples from different origins. NSPnegative sera from swine $(n=410)$, cattle $(n=175)$, and sheep $(n=61)$ were used to estimate the Dsp of each species, and NSP-positive sera from swine $(\mathrm{n}=107)$, cattle $(\mathrm{n}=70)$, and sheep $(\mathrm{n}=52)$ were used to estimate the Dsn of each species using MedCalc software.

\subsection{Comparison of accuracy rates and diagnostic performances}

The accuracy rates of 3A+3B-cCLIA and two commercial diagnostic kits were also evaluated using the abovementioned 875 NSP-negative and positive serum samples. The diagnostic performances of the four 
assays were compared by testing 430 serum samples, including 120, 60, and 77 samples from vaccinated swine, cattle, and sheep, respectively, and 173 samples from the field. In addition, serum samples from four swine challenged with FMDV O/Mya98 collected at different times were utilized to evaluate and compare the early diagnostic performance of 3B-cCLIA with 3A+3B-cCLIA and two commercial diagnostic kits.

\subsection{Estimation of the repeatability and stability of 3B-cCLIA}

Three replicates of seven serum samples with different PI values were examined on different days using plates coated in the same and different batches to calculate the intra- and inter-batch repeatability performances. After blocking, the coated plates were vacuum-packed and stored for 15 days at $37 \operatorname{deg} \mathrm{C}$ or for 1 year at 4 $\operatorname{degC}$ to identify the shelf life of the plates.

\section{RESULTS}

\subsection{Optimization of 3B-cCLIA}

The reaction conditions were optimized using checkerboard titration to obtain high ratios of N/P.

In 3B-cCLIA, the coating concentrations of $\mathrm{mAb} 2 \mathrm{G} 5$ and $3 \mathrm{ABC}$ protein were fixed at $0.25 \mu \mathrm{g} / \mathrm{mL}$, serum dilution was $1: 2.5$, concentration of 9E2-HRP was $0.005-0.02 \mu \mathrm{g} / \mathrm{mL}(0.01 \mu \mathrm{g} / \mathrm{mL})$, and reaction time was $10 \mathrm{~min}$.

\subsection{Determination of cut-off value, Dsn, and Dsp of 3B-cCLIA}

The cut-off values of 3B-cCLIA were determined using known background sera from swine (NSP-negative sera, $\mathrm{n}=410$; NSP-positive sera, $\mathrm{n}=107$ ), cattle (NSP-negative sera, $\mathrm{n}=175$; NSP-positive sera, $\mathrm{n}=$ 70) and sheep (NSP-negative sera, $\mathrm{n}=61$; NSP-positive sera, $\mathrm{n}=52$ ) by analysing the receiver operating characteristic (ROC) and interactive dot diagram (Figure 1 and Table 1). According to the ROC curve analysis, when the cut-off of 3B-cCLIA was 50\%, the Dsn was $97.20 \%(104 / 107), 95.71 \%(67 / 70)$, and 96.15\% (50/52), and Dsp was 99.51\% (408/410), 99.43\% (174/175), and 98.36\% (60/61) in swine, cattle, and sheep, respectively. Furthermore, evaluation of the cross-reaction of 3B-cCLIA with sera from other virus-infected swine (SVA, CSFV, PRRSV, PPV, and PCV2) indicated no cross-reaction (PI < 20\%).

\subsection{Accuracy rates and diagnostic performances of the four test methods}

These serum samples $(\mathrm{n}=875)$ were also tested by $3 \mathrm{~A}+3 \mathrm{~B}-\mathrm{cCLIA}$ and two commercial diagnostic kits to evaluate and compare the accuracy of the test methods (Table 1). The accuracy rate of 3B-cCLIA was almost equivalent to those of 3A+3B-cCLIA $(98.13 \%, 95.71 \%$, and $96.15 \%)$ and 3ABC-bELISA (95.33\%, $94.29 \%$, and $98.08 \%$ ) and significantly better than that of PrioCHECK FMDV NSP ELISA $(85.05 \%, 90.00 \%$, and $92.31 \%$ ) for serum samples from infected animals. For the naïve serum samples, the accuracy rate of 3B-cCLIA was equivalent to that of 3A+3B-cCLIA and slightly higher than that of 3ABC-bELISA and lower than that of PrioCHECK FMDV NSP ELISA. The accuracy rates of 3B-cCLIA and PrioCHECK FMDV NSP ELISA in testing serum samples from sows inoculated 3-15 times were almost equal to those in testing negative swine sera, and the accuracy rate of 3ABC-bELISA was slightly lower. However, the accuracy rates of the three assays in testing these sera from dairy cows inoculated 2-10 times were significantly lower than those in testing naïve cattle sera (namely, false-positive rate increase). Similarly, the accuracy rates in testing sera from sheep vaccinated 1-3 times with lab-made FMDV O, A, or Asia 1 univalent inactivated vaccine were lower than those in testing naïve sheep sera. Furthermore, false positives increased with the number of vaccinations in cattle and sheep (Table 2). To address this problem, a competitive CLIA (3A+3B-cCLIA) using $3 \mathrm{ABC}$ polyclonal antibody as the capture antibody to capture purified $3 \mathrm{ABC}$ protein and two mAbs (2G5-HRP and 9E2-HRP) as the detection antibody was developed to simultaneously detect antibodies against 3A and 3B (Figure S1). However, the accuracy rate of 3A+3B-cCLIA in testing sera from cattle and sheep vaccinated multiple times remained lower than that in testing naïve sera (Table 1). 
In addition, serum samples from 173 swine in the field were tested, and the results showed that the coincidence rates of 3B-cCLIA with 3A+3B-cCLIA, 3ABC-bELISA, and PrioCHECK FMDV NSP ELISA were 99.42\%, $96.53 \%$, and $95.37 \%$, respectively.

\subsection{Comparison of early diagnostic performance}

Seroconversion to NSP and SP was studied in four unvaccinated control swine post-challenge using O-LPBE and ID Screen ${ }^{\circledR}$ FMD Type O Competition to detect the SP antibody and using 3B-cCLIA, 3A+3B-cCLIA, 3ABC-bELISA, and PrioCHECK FMDV NSP ELISA to detect the NSP antibodies. Antibodies against SPs were first detected at $5 \mathrm{dpi}$, and all the sera examined were positive at $7 \mathrm{dpi}$ (Figure 2a). Antibodies against NSPs were detected early at 7 dpi and identified as positive in all swine at 8 dpi except for the PrioCHECK FMDV NSP ELISA (Figure 2b). Therefore, seroconversion to NSP occurred approximately 1-2 days later than that to SP.

\subsection{Repeatability and stability of 3B-cCLIA}

The assay was highly repeatable, as determined by four positive and three negative serum samples tested on different days on same and different batch plates (Figure 3). The standard positive serum (P51) and standard negative serum (P734) were tested using 3B-cCLIA plates stored for 15 days at $37{ }^{\circ} \mathrm{C}$ or for 1 year at $4{ }^{\circ} \mathrm{C}$. The $\mathrm{CL}$ values of $\mathrm{P} 734$ were $>6,000,000$, and the PI value of $\mathrm{P} 51$ was $>80 \%$. The results indicated that the 3B-cCLIA plates can be stored for up to 15 days at $37^{\circ} \mathrm{C}$ and up to 1 year at $4{ }^{\circ} \mathrm{C}$.

\section{DISCUSSION}

DIVA tests are important for serological surveillance by providing evidence of vaccinated herds infected with or freedom from FMD (W. Liu et al., 2019; Z. Z. Liu et al., 2018; Van Dreumel et al., 2015). To avoid omitting animals infected with different serotype isolates, identifying conserved mAbs is important in developing a diagnostic method for DIVA. The epitope "23 EGPYAGPLE" ${ }^{31 "}$ identified by mAb 9E2 is located in 3B and is well conserved among different serotypes of FMDV, which has been verified in a previous study (W. Liu et al., 2019). Therefore, in this study, a competitive CLIA (3B-cCLIA) using mAb 2G5 as the capture antibody to capture purified $3 \mathrm{ABC}$ protein and $9 \mathrm{E} 2-\mathrm{HRP}$ as the detection antibody was developed for DIVA. The cut-off PI value of 3B-cCLIA was determined to be $50 \%$ by testing panels of serum samples from different origins. Based on the cut-off PI of $50 \%$, diagnosis sensitivity $(97.20 \%, 95.71 \%$, and $96.15 \%)$ and diagnosis specificity $(99.51 \%, 99.43 \%$, and 98.36$)$ were determined in naïve and infected swine, cattle and sheep, respectively (Figure 1). The PrioCHECK FMDV NSP ELISA had good Dsp (accuracy rate 100\%) in testing naïve sera but had low Dsn in testing infected sera when compared with 3B-cCLIA and 3ABC-bELISA.

Although most NSPs are removed from the inactivated vaccine, residual NSPs are still present in the inactivated vaccine (W. Liu et al., 2019) and can induce the corresponding antibodies after repeated vaccination, especially in cattle (Lee, Jong, \& Yang, 2006; Mackay et al., 1998; A. K. Mohapatra et al., 2014; J. K. Mohapatra et al., 2011; Wu et al., 2011), which will interfere with the DIVA test based on detecting NSP antibodies and affect the serological surveillance and evaluation of the infection status (Fu et al., 2017). This phenomenon was also confirmed in this study, in which dairy cows vaccinated multiple times with the traditional inactivated vaccine produced a high false-positive rate using 3B-cCLIA and two commercial diagnostic kits (Tables 1 and 2). In addition, the false-positive rate in sheep vaccinated three times also increased (Tables 1 and 2). However, the false positives in sows vaccinated multiple times were almost equal to those in negative sera (Tables 1 and 2), as found in previous reports (Chung et al., 2002; Wu et al., 2011); this may be due to different immune systems in different species. False positives increased with the number of vaccinations. To address this problem, we attempted to use two mAbs recognizing NSP 3A and 3B to develop 3A+3B-cCLIA. In theory, only when the two antibodies were simultaneously present in serum, the serum was considered to be positive, thereby using the assay to try to decrease the false-positive rate in vaccinated animals. However, the results showed that $3 \mathrm{~A}+3 \mathrm{~B}-\mathrm{cCLIA}$ could not solve this false-positive 
problem (Tables 1 and 2) because $3 \mathrm{AB}_{1}, 3 \mathrm{AB}_{1} \mathrm{~B}_{2}$, and $3 \mathrm{AB}_{1} \mathrm{~B}_{2} \mathrm{~B}_{3}$ are the main forms of $3 \mathrm{~B}$-related proteins in the inactivated vaccine (data not shown) and may induce the production of corresponding antibodies.

Usually, sera from animals vaccinated multiple times were faintly positive. Therefore, it is an approach to increase the cut-off (compromise true sensitivity) to decrease the false-positive rates in testing vaccinated animals. Another approach is to establish the correlation between residual NSPs in the inactivated vaccines with different rounds of purification and the NSP antibody in different species that were inoculated different times with the corresponding inactivated vaccine, thereby conforming to a threshold value of residual NSPs in the inactivated vaccine to guide vaccine manufacturers to improve vaccine purity. In addition, the development and use of new FMD vaccines that do not contain NSP components, such as marker vaccines (Li et al., 2014; Uddowla, Hollister, Pacheco, Rodriguez, \& Rieder, 2012), virus-like particle VLP vaccines (Guo et al., 2013), multiple-epitope recombinant vaccines (Shao et al., 2011), and synthetic peptide vaccines, could eliminate this barrier.

In this study, seroconversion to NSP occurred later than that to SP, which is similar to previous reports (Mackay et al., 1998; A. K. Mohapatra et al., 2014). The seroconversion time of the PrioCHECK FMDV NSP ELISA was delayed compared with that of the other three methods, which explains the lower Dsn of the PrioCHECK FMDV NSP ELISA in detecting sera from infected animals.

In conclusion, a competitive CLIA that could rapidly detect antibodies against FMDV NSP 3B was developed in this study. 3ABC-bELISA and PrioCHECK FMDV NSP ELISA use the blocking ELISA format and need to be washed twice in process, but 3B-cCLIA uses the competitive format, only needs to be washed once and requires 15 min to obtain results. In addition, the early diagnostic performance, repeatability, and stability of 3B-cCLIA were also evaluated. These results indicated that 3B-cCLIA can be a promising tool for large-scale serological surveys in vaccinated areas for DIVA.

\section{ACKNOWLEDGMENTS}

This study was supported by grants from the National Key R\&D Program of China (2017YFD0500902 and 2016YFE0204100).

\section{CONFLICT OF INTEREST}

None of the authors has any conflicts of interest.

\section{ETHICS STAEMENT}

All of the animal experiments were conducted in accordance with the regulations for the administration of affairs concerning experimental animals approved by the State Science and Technology Commission of the People's Republic of China.

\section{REFERENCES}

Biswal, J. K., Jena, S., Mohapatra, J. K., Bisht, P., \& Pattnaik, B. (2014). Detection of antibodies specific for foot-and-mouth disease virus infection using indirect ELISA based on recombinant nonstructural protein 2B. Archives of Virology, 159 (7), 1641-1650. doi:10.1007/s00705-013-1973-3

Biswal, J. K., Ranjan, R., \& Pattnaik, B. (2016). Diagnostic application of recombinant non-structural protein 3A to detect antibodies induced by foot-and-mouth disease virus infection.Biologicals, 44 (3), 157162. doi:10.1016/j.biologicals.2016.02.004

Brocchi, E., Bergmann, I. E., Dekker, A., Paton, D. J., Sammin, D. J., Greiner, M., . . De Clercq, K. (2006). Comparative evaluation of six ELISAs for the detection of antibodies to the non-structural proteins of foot-and-mouth disease virus. Vaccine, 24 (47-48), 6966-6979. doi:10.1016/j.vaccine.2006.04.050 
Bruderer, U., Swam, H., Haas, B., Visser, N., Brocchi, E., Grazioli, S., . . Anderson, J. (2004). Differentiating infection from vaccination in foot-and-mouth-disease: evaluation of an ELISA based on recombinant 3ABC. Veterinary Microbiology, 101 (3), 187-197. doi:10.1016/j.vetmic.2004.01.021

Chung, W. B., Sorensen, K. J., Liao, P. C., Yang, P. C., \& Jong, M. H. (2002). Differentiation of foot-andmouth disease virus-infected from vaccinated pigs by enzyme-linked immunosorbent assay using nonstructural protein $3 \mathrm{AB}$ as the antigen and application to an eradication program. Journal of Clinical Microbiology, 40 (8), 2843-2848. doi:10.1128/jcm.40.8.2843-2848.2002

De Diego, M., Brocchi, E., Mackay, D., \& De Simone, F. (1997). The non-structural polyprotein 3ABC of foot-and-mouth disease virus as a diagnostic antigen in ELISA to differentiate infected from vaccinated cattle. Archives of Virology, 142 (10), 2021-2033. doi:10.1007/s007050050219

Fu, Y., Li, P., Cao, Y., Wang, N., Sun, P., Shi, Q., . . Liu, Z. (2017). Development of a Blocking ELISA Using a Monoclonal Antibody to a Dominant Epitope in Non-Structural Protein 3A of Foot-andMouth Disease Virus, as a Matching Test for a Negative-Marker Vaccine. PloS One, 12 (1), e0170560. doi:10.1371/journal.pone.0170560

Fu, Y., Lu, Z., Li, P., Cao, Y., Sun, P., Tian, M., . . . Liu, Z. (2014). Development of a blocking ELISA based on a monoclonal antibody against a predominant epitope in non-structural protein 3B2 of footand-mouth disease virus for differentiating infected from vaccinated animals. PloS One, 9 (11), e111737. doi:10.1371/journal.pone.0111737

Gao, M., Zhang, R., Li, M., Li, S., Cao, Y., Ma, B., \& Wang, J. (2012). An ELISA based on the repeated foot-and-mouth disease virus $3 \mathrm{~B}$ epitope peptide can distinguish infected and vaccinated cattle. Applied Microbiology and Biotechnology, 93 (3), 1271-1279. doi:10.1007/s00253-011-3815-0

Gao, Y., Sun, S. Q., \& Guo, H. C. (2016). Biological function of Foot-and-mouth disease virus non-structural proteins and non-coding elements. Virology Journal, 13 , 107. doi:10.1186/s12985-016-0561-z

Gelkop, S., Sobarzo, A., Brangel, P., Vincke, C., Romão, E., Fedida-Metula, S., . . L Lobel, L. (2018). The Development and Validation of a Novel Nanobody-Based Competitive ELISA for the Detection of Foot and Mouth Disease 3ABC Antibodies in Cattle. Front Vet Sci, 5 , 250. doi:10.3389/fvets.2018.00250

Guo, H. C., Sun, S. Q., Jin, Y., Yang, S. L., Wei, Y. Q., Sun, D. H., . . Liu, D. X. (2013). Foot-and-mouth disease virus-like particles produced by a SUMO fusion protein system in Escherichia coli induce potent protective immune responses in guinea pigs, swine and cattle. Veterinary Research, 44 (1), 48. doi:10.1186/12979716-44-48

Höhlich, B. J., Wiesmüller, K. H., Haas, B., Gerner, W., Correa, R., Hehnen, H. R., . . Saalmüller, A. (2003). Induction of an antigen-specific immune response and partial protection of cattle against challenge infection with foot-and-mouth disease virus (FMDV) after lipopeptide vaccination with FMDV-specific B-cell epitopes. Journal of General Virology, 84 (Pt 12), 3315-3324. doi:10.1099/vir.0.19366-0

Höhlich, B. J., Wiesmüller, K. H., Schlapp, T., Haas, B., Pfaff, E., \& Saalmüller, A. (2003). Identification of foot-and-mouth disease virus-specific linear B-cell epitopes to differentiate between infected and vaccinated cattle. Journal of Virology, 77 (16), 8633-8639. doi:10.1128/jvi.77.16.8633-8639.2003

Hosamani, M., Basagoudanavar, S. H., Tamil Selvan, R. P., Das, V., Ngangom, P., Sreenivasa, B. P., . . Venkataramanan, R. (2015). A multi-species indirect ELISA for detection of non-structural protein 3ABC specific antibodies to foot-and-mouth disease virus. Archives of Virology, 160 (4), 937-944. doi:10.1007/s00705-0152339-9

Inoue, T., Parida, S., Paton, D. J., Linchongsubongkoch, W., Mackay, D., Oh, Y., . . . Saeki, T. (2006). Development and evaluation of an indirect enzyme-linked immunosorbent assay for detection of foot-andmouth disease virus nonstructural protein antibody using a chemically synthesized 2B peptide as antigen. Journal of Veterinary Diagnostic Investigation, 18 (6), 545-552. doi:10.1177/104063870601800604 
Jaworski, J. P., Compaired, D., Trotta, M., Perez, M., Trono, K., \& Fondevila, N. (2011). Validation of an r3AB1-FMDV-NSP ELISA to distinguish between cattle infected and vaccinated with foot-and-mouth disease virus. Journal of Virological Methods, 178 (1-2), 191-200. doi:10.1016/j.jviromet.2011.09.011

Kumar, N., Sharma, R., \& Kakker, N. K. (2007). Non-structural protein 3A for differentiation of footand-mouth disease infected and vaccinated animals in Haryana (India). Zoonoses Public Health, 54 (9-10), 376-382. doi:10.1111/j.1863-2378.2007.01075.x

Lee, F., Jong, M. H., \& Yang, D. W. (2006). Presence of antibodies to non-structural proteins of foot-and-mouth disease virus in repeatedly vaccinated cattle. Veterinary Microbiology, 115 (1-3), 14-20. doi:10.1016/j.vetmic.2005.12.017

Li, P., Lu, Z., Bai, X., Li, D., Sun, P., Bao, H., . . . Liu, Z. (2014). Evaluation of a 3A-truncated footand-mouth disease virus in pigs for its potential as a marker vaccine. Veterinary Research, 45 (1), 51. doi:10.1186/1297-9716-45-51

Liu, W., Shao, J., Chen, D., Chang, Y., Chang, H., \& Zhang, Y. (2019). Identification of three linear B cell epitopes against non-structural protein 3ABC of FMDV using monoclonal antibodies. Applied Microbiology and Biotechnology, 103 (19), 8075-8086. doi:10.1007/s00253-019-10081-0

Liu, Z., Shao, J., Zhao, F., Zhou, G., Gao, S., Liu, W., . . Zhang, Y. (2017). Chemiluminescence Immunoassay for the Detection of Antibodies against the $2 \mathrm{C}$ and $3 \mathrm{ABC}$ Nonstructural Proteins Induced by Infecting Pigs with Foot-and-Mouth Disease Virus. Clinical and Vaccine Immunology, 24 (8). doi:10.1128/cvi.00153-17

Liu, Z. Z., Zhao, F. R., Gao, S. D., Shao, J. J., Zhang, Y. G., \& Chang, H. Y. (2018). Development of a chemiluminescence immunoassay using recombinant non-structural epitope-based proteins to accurately differentiate foot-and-mouth disease virus-infected and vaccinated bovines. Transboundary and Emerging Diseases, 65 (2), 338-344. doi:10.1111/tbed.12811

Lu, Z., Cao, Y., Guo, J., Qi, S., Li, D., Zhang, Q., . . . Xie, Q. (2007). Development and validation of a $3 \mathrm{ABC}$ indirect ELISA for differentiation of foot-and-mouth disease virus infected from vaccinated animals. Veterinary Microbiology, 125 (1-2), 157-169. doi:10.1016/j.vetmic.2007.05.017

Lu, Z., Zhang, X., Fu, Y., Cao, Y., Tian, M., Sun, P., . . Xie, Q. (2010). Expression of the major epitope regions of $2 \mathrm{C}$ integrated with the $3 \mathrm{AB}$ non-structural protein of foot-and-mouth disease virus and its potential for differentiating infected from vaccinated animals. Journal of Virological Methods, 170 (1-2), 128-133. doi:10.1016/j.jviromet.2010.09.016

Mackay, D. K., Forsyth, M. A., Davies, P. R., Berlinzani, A., Belsham, G. J., Flint, M., \& Ryan, M. D. (1998). Differentiating infection from vaccination in foot-and-mouth disease using a panel of recombinant, non-structural proteins in ELISA. Vaccine, 16 (5), 446-459. doi:10.1016/s0264-410x(97)00227-2

Meyer, R. F., Babcock, G. D., Newman, J. F., Burrage, T. G., Toohey, K., Lubroth, J., \& Brown, F. (1997). Baculovirus expressed $2 \mathrm{C}$ of foot-and-mouth disease virus has the potential for differentiating convalescent from vaccinated animals. Journal of Virological Methods, 65 (1), 33-43. doi:10.1016/s0166-0934(96)02165-9

Mohapatra, A. K., Mohapatra, J. K., Pandey, L. K., Sanyal, A., \& Pattnaik, B. (2014). Diagnostic potential of recombinant nonstructural protein 3B to detect antibodies induced by foot-and-mouth disease virus infection in bovines. Archives of Virology, 159 (9), 2359-2369. doi:10.1007/s00705-014-2089-0

Mohapatra, J. K., Pandey, L. K., Sanyal, A., \& Pattnaik, B. (2011). Recombinant non-structural polyprotein 3AB-based serodiagnostic strategy for FMD surveillance in bovines irrespective of vaccination. Journal of Virological Methods, 177 (2), 184-192. doi:10.1016/j.jviromet.2011.08.006

Muller, J. D., Wilkins, M., Foord, A. J., Dolezal, O., Yu, M., Heine, H. G., \& Wang, L. F. (2010). Improvement of a recombinant antibody-based serological assay for foot-and-mouth disease virus. Journal of Immunological Methods, 352 (1-2), 81-88. doi:10.1016/j.jim.2009.11.004 
Oem, J. K., Chang, B. S., Joo, H. D., Yang, M. Y., Kim, G. J., Park, J. Y., . . Joo, Y. S. (2007). Development of an epitope-blocking-enzyme-linked immunosorbent assay to differentiate between animals infected with and vaccinated against foot-and-mouth disease virus. Journal of Virological Methods, 142 (1-2), 174-181. doi:10.1016/j.jviromet.2007.01.025

Oem, J. K., Kye, S. J., Lee, K. N., Park, J. H., Kim, Y. J., Song, H. J., \& Yeh, M. (2005). Development of synthetic peptide ELISA based on nonstructural protein $2 \mathrm{C}$ of foot and mouth disease virus. Journal of Veterinary Science, 6 (4), 317-325.

Robiolo, B., Seki, C., Fondevilla, N., Grigera, P., Scodeller, E., Periolo, O., . . Mattion, N. (2006). Analysis of the immune response to FMDV structural and non-structural proteins in cattle in Argentina by the combined use of liquid phase and 3ABC-ELISA tests. Vaccine, 24 (7), 997-1008. doi:10.1016/j.vaccine.2005.08.071

Shao, J. J., Wong, C. K., Lin, T., Lee, S. K., Cong, G. Z., Sin, F. W., . . . Liu, J. X. (2011). Promising multiple-epitope recombinant vaccine against foot-and-mouth disease virus type $\mathrm{O}$ in swine. Clinical and Vaccine Immunology, 18 (1), 143-149. doi:10.1128/cvi.00236-10

Sharma, G. K., Mohapatra, J. K., Pandey, L. K., Mahajan, S., Mathapati, B. S., Sanyal, A., \& Pattnaik, B. (2012). Immunodiagnosis of foot-and-mouth disease using mutated recombinant 3ABC polyprotein in a competitive ELISA. Journal of Virological Methods, 185 (1), 52-60. doi:10.1016/j.jviromet.2012.05.029

Shen, F., Chen, P. D., Walfield, A. M., Ye, J., House, J., Brown, F., \& Wang, C. Y. (1999). Differentiation of convalescent animals from those vaccinated against foot-and-mouth disease by a peptide ELISA. Vaccine, 17 (23-24), 3039-3049. doi:10.1016/s0264-410x(99)00148-6

Sørensen, K. J., de Stricker, K., Dyrting, K. C., Grazioli, S., \& Haas, B. (2005). Differentiation of foot-andmouth disease virus infected animals from vaccinated animals using a blocking ELISA based on baculovirus expressed FMDV 3ABC antigen and a 3ABC monoclonal antibody.Archives of Virology, 150 (4), 805-814. doi:10.1007/s00705-004-0455-Z

Sørensen, K. J., Madsen, K. G., Madsen, E. S., Salt, J. S., Nqindi, J., \& Mackay, D. K. (1998). Differentiation of infection from vaccination in foot-and-mouth disease by the detection of antibodies to the non-structural proteins 3D, 3AB and 3ABC in ELISA using antigens expressed in baculovirus. Archives of Virology, 143 (8), 1461-1476. doi:10.1007/s007050050390

Uddowla, S., Hollister, J., Pacheco, J. M., Rodriguez, L. L., \& Rieder, E. (2012). A safe foot-and-mouth disease vaccine platform with two negative markers for differentiating infected from vaccinated animals. Journal of Virology, 86 (21), 11675-11685. doi:10.1128/jvi.01254-12

Uttenthal, A., Parida, S., Rasmussen, T. B., Paton, D. J., Haas, B., \& Dundon, W. G. (2010). Strategies for differentiating infection in vaccinated animals (DIVA) for foot-and-mouth disease, classical swine fever and avian influenza. Expert Rev Vaccines, 9 (1), 73-87. doi:10.1586/erv.09.130

Van Dreumel, A. K., Michalski, W. P., McNabb, L. M., Shiell, B. J., Singanallur, N. B., \& Peck, G. R. (2015). Pan-Serotype Diagnostic for Foot-and-Mouth Disease Using the Consensus Antigen of Nonstructural Protein 3B. Journal of Clinical Microbiology, 53 (6), 1797-1805. doi:10.1128/jcm.03491-14

Wang, C., Jie, W. U., Zong, C., Jie, X. U., \& Huang-Xian, J. U. (2012). Chemiluminescent Immunoassay and Its Applications. Chinese Journal of Analytical Chemistry, 40 (1), 3-10.

Wu, L., Jiang, T., Lu, Z. J., Yang, Y. M., Sun, P., Liang, Z., . . . Liu, Z. X. (2011). Development and validation of a prokaryotically expressed foot-and-mouth disease virus non-structural protein $2 \mathrm{C}^{\prime} 3 \mathrm{AB}$-based immunochromatographic strip to differentiate between infected and vaccinated animals. Virology Journal, 8 , 186. doi:10.1186/1743-422x-8-186

Figure 1. Receiver operating characteristic (ROC) analysis for the determination of the cut-off value of 3BcCLIA. (A, C, E) Interactive dot diagram of 3B-cCLIA in testing sera from swine, cattle, and sheep. 0, negative serum samples $(\mathrm{n}=410,175$, and 61$) ; 1$, positive serum samples $(\mathrm{n}=107,70$, and 52$)$. (B, $\mathrm{D}$, 
and F) Each point on the ROC curve represents a sensitivity-specificity pair in testing the sera from swine, cattle, and sheep.

Figure 2. Early production of antibodies against FMDV SPs and NSPs in sera from experimentally challenged swine. (A) Thirty-two serum samples from four unvaccinated control swine after challenge with FMDV O/Mya98 were collected at 0 dpi and 2-8 dpi and tested using O-LPBE, ID Screenß FMD Type O Competition to detect SP antibodies. The dashed lines _ _ _ and ...... represent the cut-off values of O-LPBE and ID Screen ${ }^{\circledR}$ FMD Type O Competition, respectively. (B) Thirty-two serum samples were tested using 3BcCLIA, 3A+3B-cCLIA, and two commercial diagnostic kits (3ABC-bELISA and PrioCHECK FMDV NSP ELISA) to detect NSP antibodies. The dashed line ( $\left.\ldots+-)_{-}\right)$represents the cut-off value of 3B-cCLIA, 3ABC-bELISA, and PrioCHECK FMDV NSP ELISA, and the dashed line (.....) represents the cut-off value of $3 \mathrm{~A}+3 \mathrm{~B}-\mathrm{cCLIA}$. The bar represents the range of values obtained by detecting sera from four swine at the given time.

Figure 3. Intra- and inter-batch repeatability performances of 3B-cCLIA were assessed using four positive and three negative serum samples tested on different days on same and different batch plates. The dashed line represents the cut-off value of 3B-cCLIA.

\section{Hosted file}

Table 1-2.pdf available at https://authorea.com/users/391397/articles/505517-development-ofa-competitive-chemiluminescence-immunoassay-using-a-monoclonal-antibody-recognizing-3bof-foot-and-mouth-disease-virus-for-differentiating-infected-from-vaccinated-animals 
A$$
\text { A }
$$

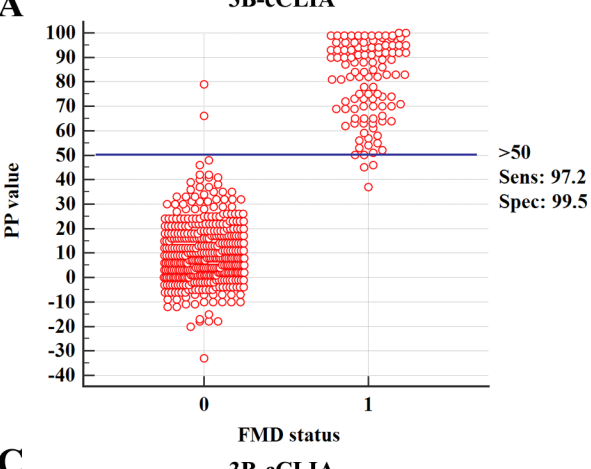

C

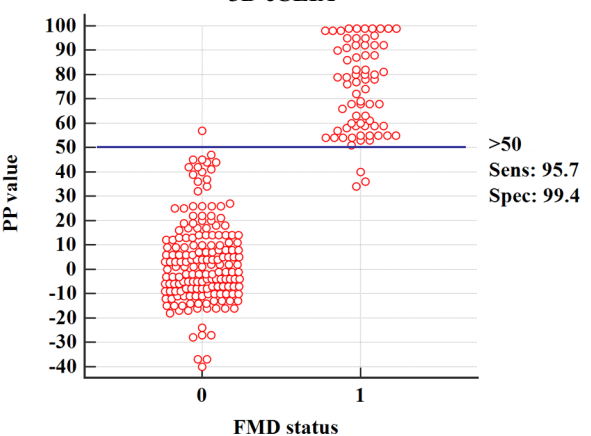

E

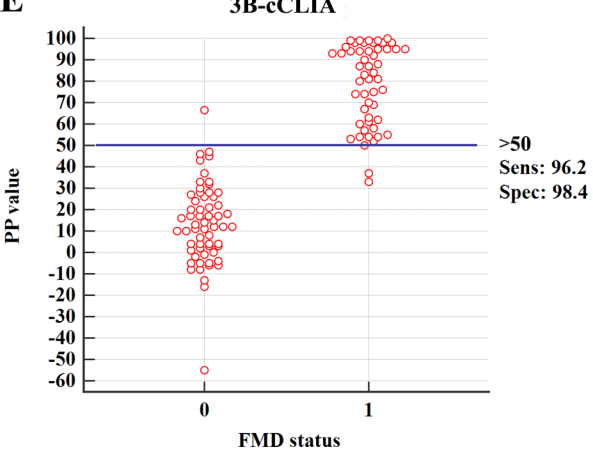

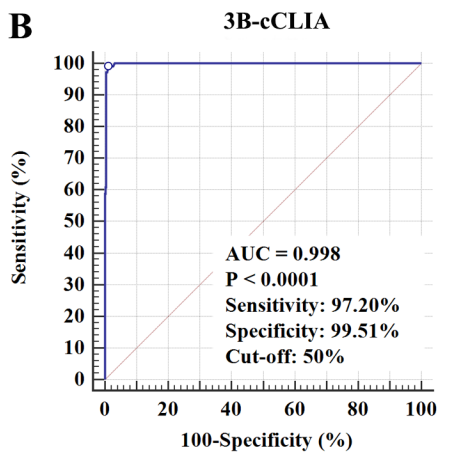

D

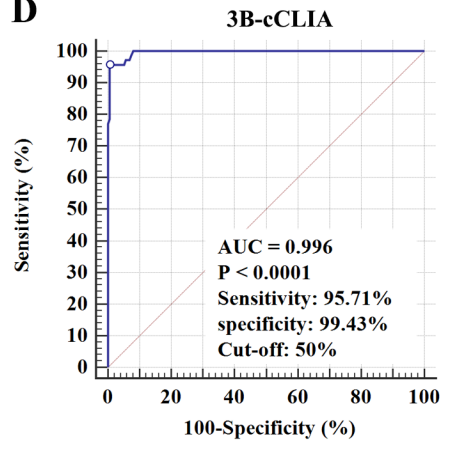

F

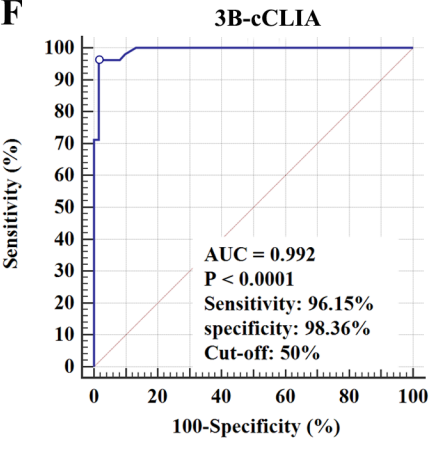

A

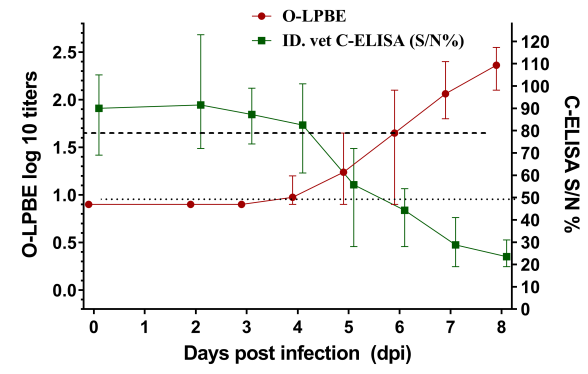

B

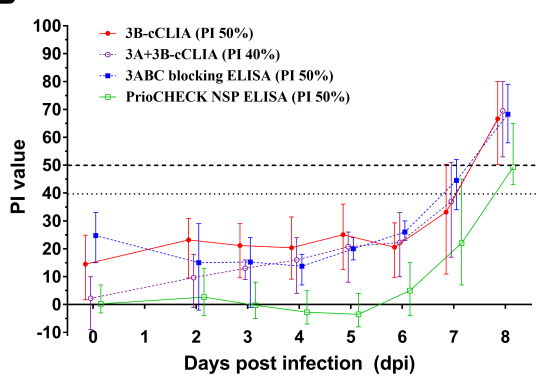




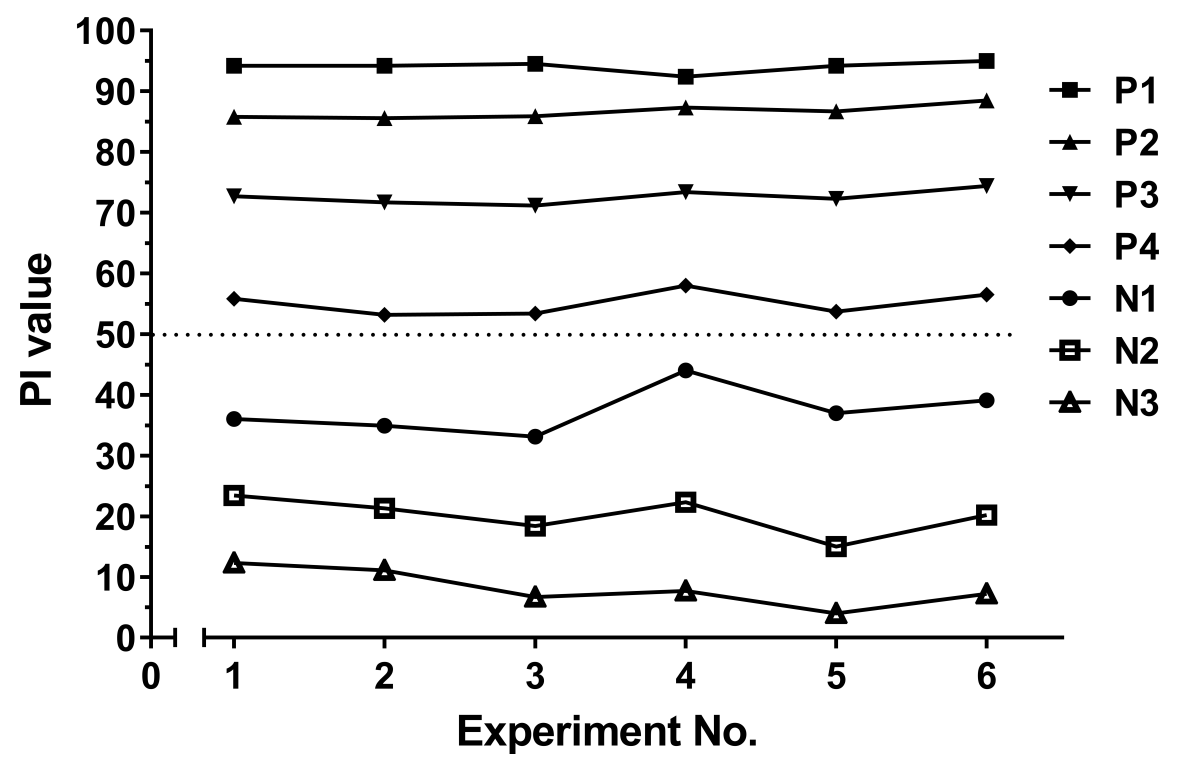

